\title{
Plasmodium falciparum msp2 Genotypes and Multiplicity of Infections among Children under Five Years with Uncomplicated Malaria in Kibaha, Tanzania
}

\author{
W. Kidima and G. Nkwengulila \\ College of Natural and Applied Sciences, Department of Zoology and Wildlife Conservation, University of Dar es Salaam, \\ P.O. Box 35064, Dar es Salaam, Tanzania
}

Correspondence should be addressed to W. Kidima; kidimaw@udsm.ac.tz

Received 11 August 2015; Revised 17 November 2015; Accepted 19 November 2015

Academic Editor: José F. Silveira

Copyright (C) 2015 W. Kidima and G. Nkwengulila. This is an open access article distributed under the Creative Commons Attribution License, which permits unrestricted use, distribution, and reproduction in any medium, provided the original work is properly cited.

\begin{abstract}
Genetic diversity of Plasmodium falciparum may pose challenges in malaria treatment and prevention through chemotherapy and vaccination. We assessed Plasmodium falciparum genetic diversity and multiplicity of infection (MOI) of $P$. falciparum infections and sort relationship of parasitaemia with P. falciparum msp2 genotypes as well as with the number of infecting clones. The study was carried out in Kibaha, Tanzania. Ninety-nine children under five years with uncomplicated malaria were recruited. Genetic diversity was analyzed by genotyping the $m s p 2$ gene using PCR-Restriction Fragment Length Polymorphism. Thirty-two different $m s p 2$ alleles were obtained. The $m s p 23 \mathrm{D} 7$ allelic frequency was higher $(48.1 \%)$ and more prevalent than FC27 (27.3\%) $(p<0.05)$. Twenty-four percent of the infections were mixed alleles. The individuals with FC27 had high parasitemia compared to those with 3D7 alleles $(p=0.038)$. The mean MOI was low (1.4 clones, 95\% CI 1.2-1.5). The P. falciparum population among children at Kibaha is composed of distinct $P$. falciparum clones, and parasites having 3D7 are more frequent than those with FC27 alleles. Individuals with parasite having FC27 alleles have high parasite densities suggesting that parasites with FC27 alleles may associate with severity of disease in Kibaha. Low MOI at Kibaha suggests low malaria transmission rate.
\end{abstract}

\section{Introduction}

Genetic diversity within P. falciparum is a major characteristic and a factor by which the parasites survive the hosts' immune responses. It results from allelic polymorphism, recombination, chromosome rearrangements, and antigenic variation [1]. Experimental crossing of genetically distinct cloned lines of this species by Walliker et al. (1987) [2] established that genetic recombination occurs during meiosis, creating parasites of novel genotypes. Genetic diversity of malaria parasites represents a major issue in understanding several aspects of malaria infection and disease-transmission dynamics and has bearing on malaria vaccine development. In endemic areas, individuals are continuously exposed to infective mosquito bites. Due to high malaria transmission intensity in some parts of Tanzania, an individual may be infected with genetically diverse clones that may be related to the risk of clinical malaria [3-6]. It is thus imperative to assess diversity of strains/clones in blood samples among individuals in malarial areas.

The mechanisms controlling the genetic diversity within the parasite genome are many and complex. Studies have shown extensive polymorphism in some key parasite antigen during the parasites asexual blood stage (reviewed in [7]). The extensive degree of polymorphism noted in many surface antigens contributes to immune evasion and aids parasite pathogenesis [8]. It has been proposed that polymorphism of blocks 2 and 3 of the gene coding for the $m s p 1, m s p 2$, and glurp of $P$. falciparum can be used as genetic markers for genotyping of field parasite populations [9]. Studies have shown that the locus of the $m s p 2$ gene of $P$. falciparum is extremely polymorphic [10] and therefore most informative. Understanding genetic diversity and multiplicity of infection is important in understanding the epidemiology of Plasmodium falciparum infections in endemic areas. 


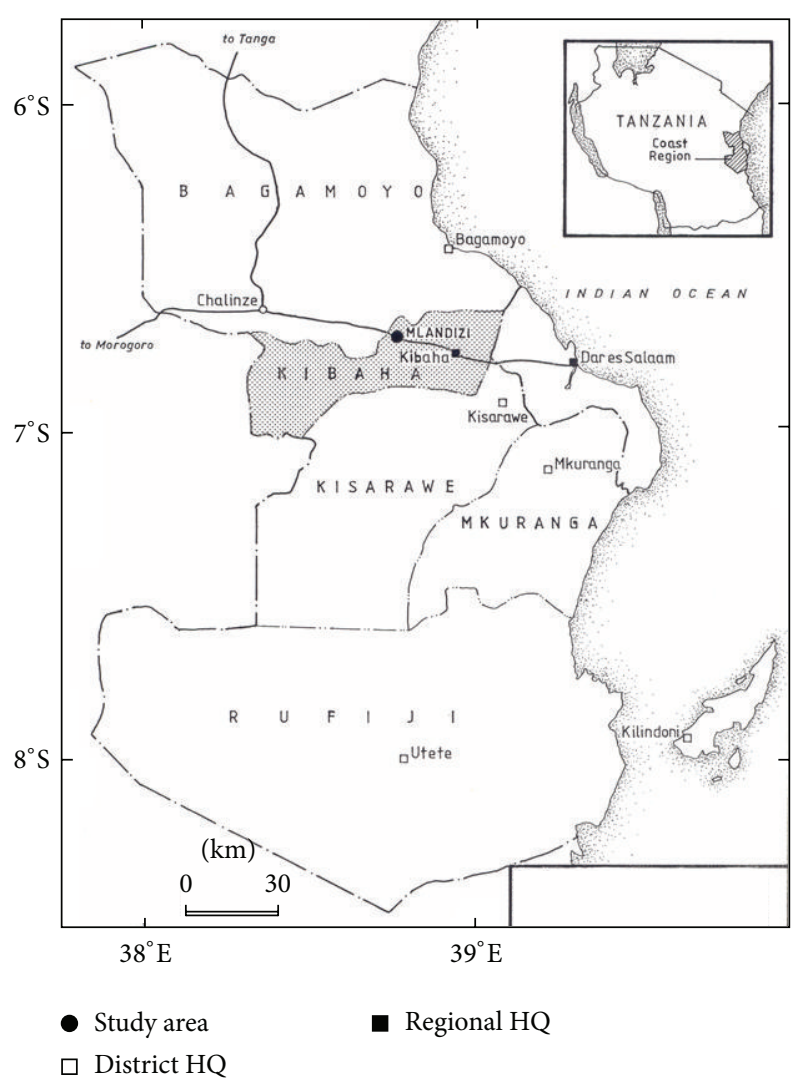

FIgURe 1: Location of the study area.

In the present study, Polymerase Chain Reaction- (PCR-) Restriction Fragment Length Polymorphism (RFLP) was used to define the diversity and distribution of $m s p 2$ of alleles among symptomatic $P$. falciparum infections and assess the multiplicity of infection among children with uncomplicated malaria. The $m s p 2$ genotypes and multiplicity of infection were related to peripheral parasitaemia.

\section{Materials and Methods}

This study was carried out at Mlandizi, Kibaha, in the Coastal Region, Tanzania (Figure 1). Mlandizi was an ideal site for this study, as malaria transmission is perennial with a mean annual entomological inoculation rate of over 200 infective bites per person per year [11]. In this region, $P$. falciparum is the dominant malaria species. Malaria transmission in Kibaha peaks towards the end of the long and short rains.

2.1. Blood Sample Collection. Plasmodium falciparum isolates were collected during a monitoring programme of Sulphadoxine-Pyrimethamine (SP) to establish its efficacy using WHO 14-day protocol. One hundred and nineteen (119) children aged 6-59 months attending Mlandizi Health Centre were enrolled during the 2002 rainy season. Inclusion criteria were monoinfection with $P$. falciparum with at least 300 parasites $/ \mu \mathrm{L}$, no intake of antimalarials or sulphonamidebased drugs during the previous 4 weeks, absence of severe illness, presence of axillary temperature of $\geq 37.5^{\circ} \mathrm{C}$ but $<39.5^{\circ}$, no history of allergic reactions to the administered drug, and willingness to give consent to participate. Infected blood was taken from children presenting with nonsevere malaria at day 0 , before drug administration. Upon presentation to day 0 , thick and thin blood smears were collected by finger prick and stained with Giemsa for microscopic parasite identification and quantification. In the thick smear, parasites were counted against 200 white blood cell count, assuming this to be $8000 / \mu \mathrm{L}$. Parasite density was recorded as the number of parasites per 200 white blood cells (WBC). Taking $8000 \mathrm{WBC}$ as the conversion factor, densities were converted to the number of parasites/ $\mu \mathrm{L}$ of blood. The parasites density was expressed as the number of parasites $/ \mu \mathrm{L}$. Assessment of the treatment response was based on the WHO 14-day in vivo protocol.

Ethical approval was given by the National Institute for Medical Research, clearance ref. no. NIMR/HQ/R.8a/ Vol.1X/107. Verbal informed consent was obtained from village leaders and written consent was obtained from all parents or guardians.

2.2. DNA Extraction and Genotyping of msp2 Gene. At enrolment, finger prick blood was also spotted on $3 \mathrm{~mm}$ Whatman filter paper (Whatman International Ltd., Maidstone, United Kingdom), dried, and kept separately to avoid contamination at room temperature. Parasite genomic DNA was extracted from 116 samples by chelex extraction as described elsewhere [12]. Briefly, sample parasites were boiled in the presence of chelex, heavy metal chelator. The blotted filter paper of about $3 \mathrm{~mm}^{2}$ was cut and placed in $1.5 \mathrm{~mL}$ capacity microfuge tubes containing $1 \mathrm{~mL}$ of PBS (pH 7.4) and $50 \mu \mathrm{L}$ of $10 \%$ saponin and incubated at $4^{\circ} \mathrm{C}$ overnight. The tubes were then centrifuged to aspirate the reddish PBS/saponin. Thereafter, $1 \mathrm{~mL}$ of PBS was added to each tube inverted each time and then incubated at $4^{\circ} \mathrm{C}$ for two hours. The solution was then centrifuged for two minutes and again the aspiration of the liquid was done, drying the filter paper by removing the liquid. $100 \mu \mathrm{L}$ of sterile water was added to each tube together with $50 \mu \mathrm{L}$ of $20 \%$ chelex. Chelex was then transferred to the microfuge tube using a yellow tip, with its tapered end clipped off, inverting the chelex solution after every transfer. Then, the parasite DNA was extracted by incubating the tubes for 20 minutes on a $100^{\circ} \mathrm{C}$ heat block vigorously vortexing each sample every two minutes.

After incubation, tubes were centrifuged for 2 minutes and then 10 minutes at 14,000 rotations per minute to remove chelex resin, leaving the parasite DNA in the supernatant. The parasite DNA was then stored in microfuge tubes at $-20^{\circ} \mathrm{C}$, for PCR amplification. To genotype different forms of $P$. falciparum in these samples, the polymorphic repetitive regions of the merozoite surface protein 2 (msp2) were amplified by a nested PCR assay [13] using oligonucleotide primer pairs specific for $P$. falciparum and alleles determined by RFLP. The initial amplification using the outermost $5^{\prime}$ and $3^{\prime}$ primers, S2, and S3 (Table 1) was carried out on $20 \mu \mathrm{L}$ reaction volume containing Ix PCR Buffer- $\mathrm{MgCl}_{2}$, (Invitrogen), $1.5 \mathrm{mM} \mathrm{MgCl}, 125 \mu \mathrm{M}$ mixture of deoxynucleoside 
TABle 1: Sequence of primers for amplifying msp2 gene of Plasmodium falciparum.

\begin{tabular}{llc}
\hline Primer & Sequence & Primer pair \\
\hline S2 & $5^{\prime}$-GAA GGT AAT TAA AAC ATT GTC-3' ${ }^{\prime}$ (sense) & S2/S3 \\
S3 & $5^{\prime}$-GAG GGA TGT TGC TGC TCC ACA-3' (antisense) & S1/S4 \\
\hline S1 & $5^{\prime}$-GAG TAT AAG GAG AAG TAT G-3 ${ }^{\prime}$ (sense) & \\
S4 & $5^{\prime}$-CTA GAA CCA TGC ATA TGT CC-3' (antisense) & \\
\hline
\end{tabular}

phosphates, $0.25 \mu \mathrm{M}$ of primer pair (S2/S3), $0.02 \mathrm{U}$ of Taq DNA polymerase, and $5 \mu \mathrm{L}$ of genomic DNA. The PCR reaction involved 30 amplification cycles composed of initial denaturation at $94^{\circ} \mathrm{C}$ for 5 minutes, a denaturing step at $94^{\circ} \mathrm{C}$ for 1 minute, followed by annealing at $55^{\circ} \mathrm{C}$ for 2 minutes, and an extension step for 2 minutes at $72^{\circ} \mathrm{C}$. In the second round amplification reaction, $2 \mu \mathrm{L}$ of DNA amplicon from the primary PCR was used as a template in a $30 \mu \mathrm{L}$ reaction volume containing same reaction mix as in the primary reaction, using nested conserved region $5^{\prime}$ and $3^{\prime}$ primers $S 1$ and S4 (Table 1) to amplify the central region of $m s p 2$ gene. Same cycling parameters were used as in the primary PCR. The nested PCR products were then monitored on $2 \%$ agarose gel before restriction digestion. A Hinf I (New England Biolabs) restriction digest was performed to identify $m s p 2$ alleles in parasite isolates. Digestion was carried out overnight and digestion products were run on $10 \%$ PAA gel (Q-Bio gene, Canada) using $1.5 \mathrm{~mm}$ spacers, stained with ethidium bromide, then observed under UV light, and photographed. A $1 \mathrm{~Kb}$ ladder was used as a DNA size was used as a marker on all gels. Hinf I restriction digestion of a nested PCR product of FC27-type alleles produced two conserved fragments of 115 and 137 base pairs (bp) and a repeat unit of $96 \mathrm{bp}$, which represent the $5^{\prime}$ and $3^{\prime}$ end of amplification product, respectively. Members of the 3D7-type alleles produced two conserved restriction fragments of 70 and $108 \mathrm{bp}$ in length [13].

2.3. Data Analysis. Shannon winner index was used to calculate diversity index of $m s p 2$ alleles. To analyze differences in the frequency of occurrence of 3D7 and FC27 alleles in sample isolates, $Z$ score statistical test was used. Twoway ANOVA was used to compare mean parasite densities between children with FC27, 3D7, and mixed allele using Prism 5 (Graph Pad software). Multiplicity of infection was calculated as the highest number of alleles of each $P$. falciparum isolate.

\section{Results}

3.1. msp2 Genotypes of Plasmodium Falciparum. To assess P. falciparum msp2 genotypes 99 parasite isolate (blood samples) were analysed. Polymerase chain reaction analysis showed that (82) $81 \%$ samples were positive for P. falciparum. All 82 positive samples were subjected to RFLP analysis to define $m s p 2$ genotypes/multiple infections in parasite isolates. The Hinf I enzyme resulted in genotype-specific pattern of bands that allow discriminating a multitude of different msp2 alleles. Individual alleles were named according to

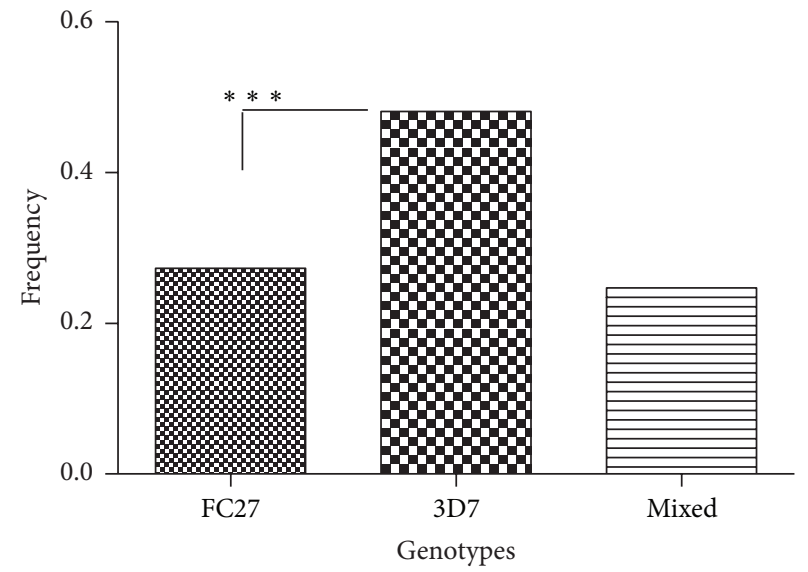

FIGURE 2: Frequency of occurrence of $m s p 2$ genotypes among children under five years at Kibaha $(N=83)\left({ }^{* * *}\right.$ significant difference, $p<0.05, Z$-score test).

the size of a further large Hinf I fragment, which varies between alleles.

The $m s p 2$ alleles were grouped according to similarities in their PCR-RFLP patterns. The frequency of occurrence of the two $m s p 2$ genotypes is shown in Figure 2. Most children were infected by parasites of the 3D7 allelic family; consequently the distribution of $m s p 2$ genotypes in different age groups showed that the 3D7 allelic types were the most prevalent in each age group (Figure 3). Overall, 51 different $m s p 2$ alleles were detected by Hinf I restriction digestion of a nested PCR product. Fifteen $m s p 2$ alleles belonged to FC27 and 17 alleles belonged to 3D7 alleles and 19 were mixed from both 3D7 and FC27 (Table 2).

There were significant differences between mean parasite densities between individuals with FC27, 3D7, and mixed genotypes (ANOVA, $p=0.0389$ ) with high parasite densities in children carrying $P$. falciparum parasites belonging to the FC27 alleles (Figure 4). Allele diversity was calculated by Shannon wiener indices $\left(H^{\prime}\right)$. The diversity of FC27 allele was higher $\left(H^{\prime}=1.15\right)$ than that of $3 \mathrm{D} 7\left(H^{\prime}=1.08\right)$ but the difference was not statistically significant (Special $t$-test, $\mathrm{df}=$ $53, p>0.05)$.

3.2. Multiplicity of Infection (MOI). The multiplicity of infection represents the actual number of coinfecting genotypes circulating in an individual. It was calculated as the highest number of alleles of each P. falciparum isolate. Most children were infected by at least two distinct parasites genotypes. Fifty percent $(n=38)$ of infected blood samples contained single 
TABLE 2: The 3D7 and FC27 P. falciparum msp2 alleles recorded from children less than five years at Kibaha.

\begin{tabular}{lccc}
\hline 3D7 alleles & Frequency & FC27 alleles & Frequency \\
\hline 3D7 (200), (270) & 1 & FC27 (270) & 2 \\
3D7 (390) & 2 & FC27 (500) & 1 \\
3D7 (400) & 4 & FC27 (450) & 1 \\
3D7 (370) & 9 & FC27 (350) & 1 \\
3D7 (350) & 6 & FC27 (290) & 2 \\
3D7 (450) & 2 & FC27 (170) & 2 \\
3D7 (360), (400) & 2 & FC27 (250) & 1 \\
3D7 (470) & 1 & FC27 (330) & 1 \\
3D7 (320) & 1 & FC27 (137), (170) & 2 \\
3D7 (320) & 1 & FC27 (150), (170) & 1 \\
3D7 (300), (400) & 2 & FC27 (170) & 1 \\
3D7 (210) & 1 & FC27 (170), (260) & 1 \\
3D7 (440) & 1 & FC27 (150), (290) & 2 \\
3D7 (250), (400) & 1 & FC27 (137) & 2 \\
3D7 (520) & 1 & FC27 (290) & 1 \\
3D7 (170), (260) & 1 & & \\
3D7 (370), (500) & 1 & & \\
\hline
\end{tabular}

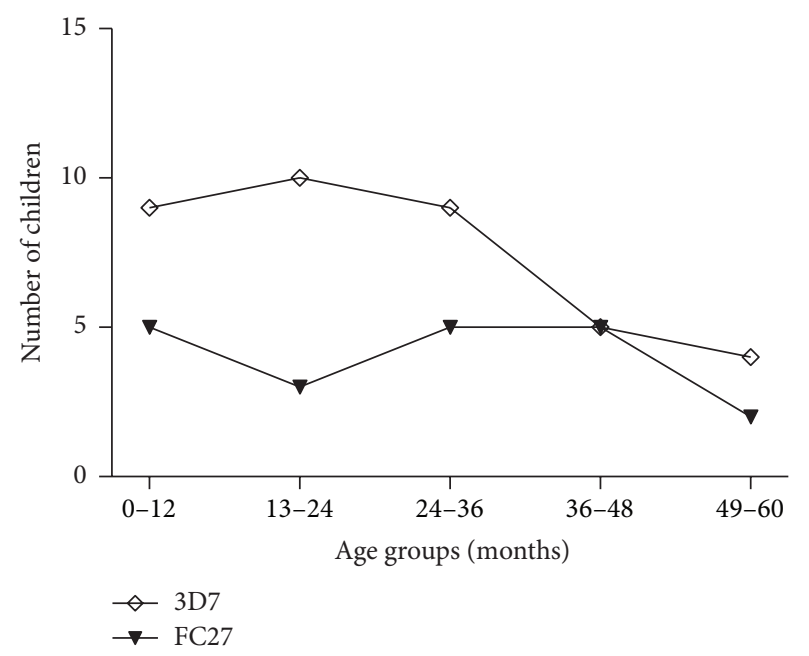

Figure 3: Distribution of $m s p 2$ genotypes among children of different age groups at Kibaha at baseline $(N=65)$.

infection, 47\% $(n=36)$ double infections, and 3\% $(n=2)$ triple concurrently infecting genotypes. The mean number of concurrent infections (i.e., MOI) was 1.4 (95\% CI 1.2-1.5) (Figure 5). The highest MOI of 1.6 was observed amongst the 2 -year-old children. There was significant difference between $\mathrm{MOI}$ in the youngest children (0-12 months) and MOI found in other age groups ( $t$-test, $p \leq 0.05)$.

\section{Discussion}

The analysis of malaria-infected blood revealed by PCRRFLP in our study has shown considerable heterogeneity among $P$. falciparum infections. We found 51 different $m s p 2$ alleles of $P$. falciparum-msp 2 gene in blood samples collected

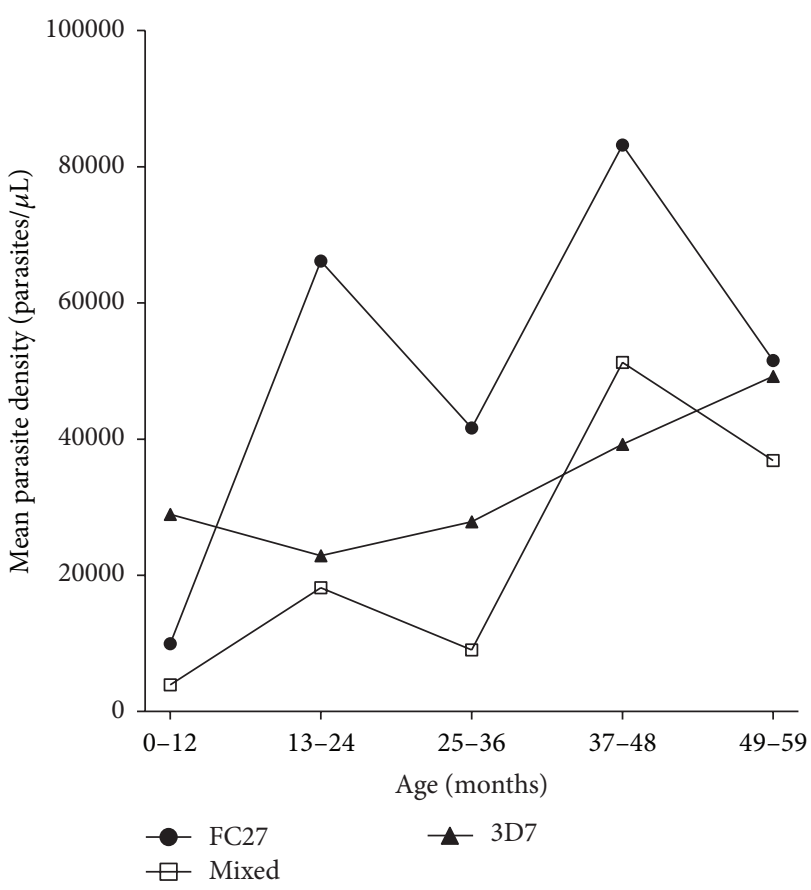

FIGURE 4: Relationship between mean parasite density and parasite genotypes in each age group $(N=83)$. Significant difference between mean parasite densities (ANOVA $F(2,4)=4.2, p=0.0389$ ).

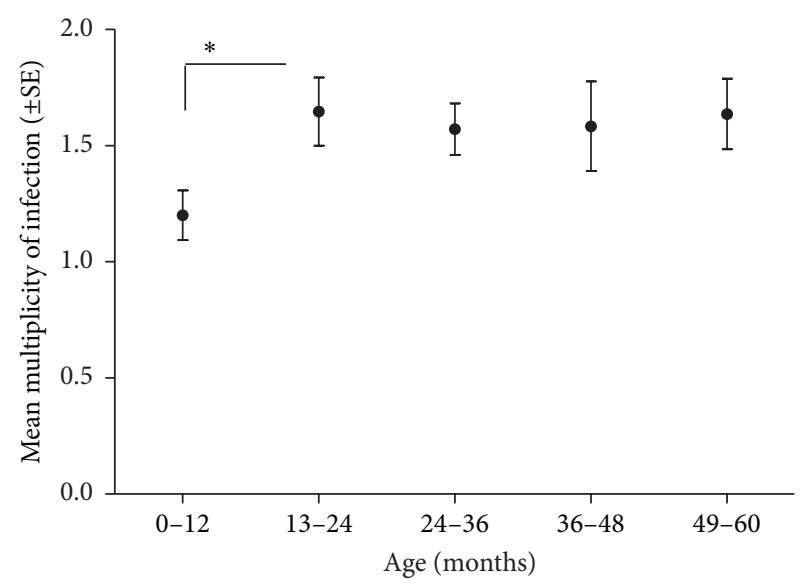

Figure 5: Multiplicity of Plasmodium falciparum infections in relation to child age at Kibaha at day $0\left(N=83, t\right.$-test, $\left.{ }^{*} p \leq 0.02\right)$.

from children under five years with clinical malaria. In this study, P. falciparum harboring the 3D7 parasites were more frequent than the FC27 parasites in blood samples collected from children under five years with uncomplicated malaria in Kibaha. Our findings are in agreement with other studies carried out in children with uncomplicated malaria in various malaria endemic areas $[14,15]$, suggesting that the 3D7 parasite strain may be the common genotype circulating in malaria endemic areas.

Although the FC27 parasites were less frequent in children under five years in Kibaha, children having these parasites genotypes had higher mean parasite density $(p=0.038)$. 
Association of FC27 parasites with high parasite density may suggest lack of antigen-specific antibodies amongst children due to lack of exposure and parasite immunosuppressive effects $[16,17]$, the conditions which might have allowed multiplication of parasites at higher densities in these infected individuals. Soulama et al. 2009 [18] observed high frequency of parasites having FC27 allelic family in severe malaria with and without anemia in children less than 5 years old in Burkina Faso, implying that the FC27 might be associated with severity of disease. Further studies in other endemic areas have to be carried out to confirm these observations.

The overall mean MOI observed in Kibaha, Mlandizi, is lower (1.4 parasite clones) compared to other holoendemic areas including some parts of Tanzania. Felger et al. (1999) [13] reported MOI of 4 at Kilombero, Tanzania, and 9.4 in Muheza, Tanga [4]. In Senegal, MOI was reported as 5 [19] and 2.1 in Kenya [20,21]. The spatial variability in the mean MOI in malaria endemic areas may be attributed to variations in ecological parameters and anthropogenic factors. In Kibaha, being a semiurban area, transmission intensity may be low because of the lower density of Anopheles vectors. Another reason contributing to the low MOI was pointed out by Contamin et al. (1995) [22] and Magesa (1999) [4], that the effect might be an artifact caused by a single dominant clone in clinical cases overshadowing the PCR template occurring at low density. This could result in identifying few genotypes despite the presence of other genotypes in the sample. The lowest MOI in Mlandizi was observed in the youngest children (0-12). This may most probably be attributed to the short period of exposure to infection that the youngest children have had. Also protection from maternal immunity, which might have restricted the development of some genotypes, cannot be discounted.

There was a weak-negative association between MOI and parasite density but the relationship was not significant (see Figure 6). The weak association between MOI and parasite density might stem from the small sample size used in this study. However, the trend of low MOI in isolates with higher parasite densities suggests multiplication of a single dominant clone in the absence of high competition. Such trend has also been observed in children suffering from severe malaria in Dakar by Robert et al. (1996) [23]. Recent studies by Rono et al. 2013 [24] have implicated higher MOI with protection against severe disease in children under five, further demonstrating the role of MOI in clinical malaria.

\section{Conclusion}

We have demonstrated that the genetic diversity of $P$. falciparum isolates from children under five years at Kibaha is extensively illustrated by greater polymorphism that exists in the parasite $m s p 2$ gene. In addition, we found that the 3D7 genotypes are more frequent than the FC27 genotypes in children under five at Kibaha. However, the FC27 genotypes are associated with higher parasite densities in children of $1 \leq 4$ years in Kibaha. Our data are important in understanding the molecular epidemiology of $P$. falciparum malaria in Kibaha, indirectly demonstrating the immune status of children with

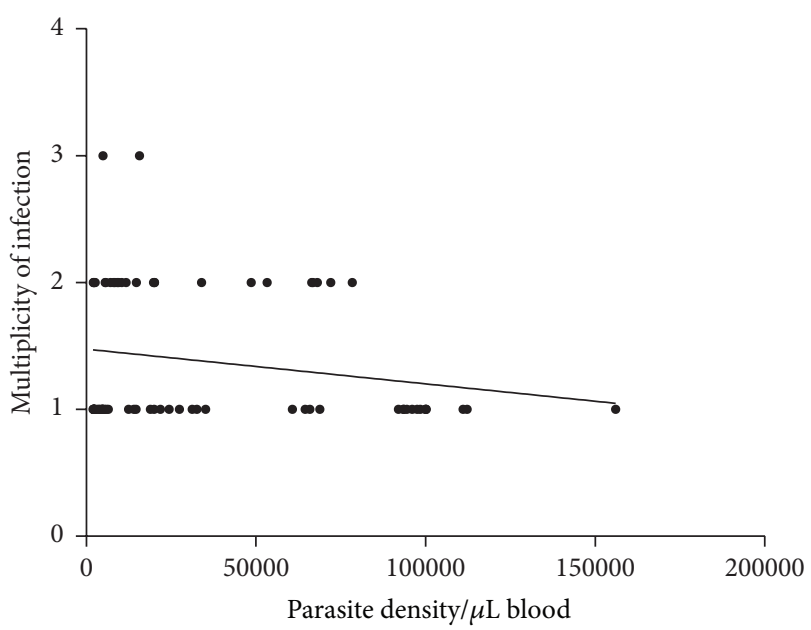

FIGURE 6: Relationship between multiplicity of infection and parasite density. Spearman rank correlation test $(r=-0.056$, CI $95 \%-0.2840$ to 0.1785 ).

uncomplicated malaria and transmission intensity of malaria, which is spatially different in endemic areas.

\section{Conflict of Interests}

The authors declare that there is no conflict of interests regarding the publication of this paper.

\section{Acknowledgments}

The authors would like to give sincere thanks to Professor Zul Premji, Christopher Membi, Dr. Hassan Mshinda, and the Molecular Parasitology group at Ifakara Health Institute, for their technical assistance. This study received financial assistance from DAAD.

\section{References}

[1] D. J. Kemp, A. F. Cowman, and D. Walliker, "Genetic diversity in Plasmodium falciparum," Advances in Parasitology, vol. 29, pp. 75-149, 1990.

[2] D. Walliker, I. A. Quakyi, T. E. Wellems et al., "Genetic analysis of the human malaria parasite Plasmodium falciparum," Science, vol. 236, no. 4809, pp. 1661-1666, 1987.

[3] F. Al-Yaman, B. Genton, J. C. Reeder, R. F. Anders, T. Smith, and M. P. Alpers, "Reduced risk of clinical malaria in children infected with multiple clones of Plasmodium falciparum in a highly endemic area: a prospective community study," Transactions of the Royal Society of Tropical Medicine and Hygiene, vol. 91, no. 5, pp. 602-605, 1997.

[4] S. M. Magesa, Malaria parasite dynamics: epidemiology, allelic diversity and turnover rates of Plasmodium falciparum infections in Tanzania children [Ph.D. thesis], University of Copenhagen, Copenhagen, Denmark, 1999.

[5] S. Bereczky, A. Liljander, I. Rooth et al., "Multiclonal asymptomatic Plasmodium falciparum infections predict a reduced risk of malaria disease in a Tanzanian population," Microbes and Infection, vol. 9, no. 1, pp. 103-110, 2007. 
[6] A. Färnert, T. N. Williams, T. W. Mwangi et al., "Transmissiondependent tolerance to multidonal Plasmodium falciparum infection," Journal of Infectious Diseases, vol. 200, no. 7, pp. 11661175, 2009.

[7] A. Bolad and K. Berzins, "Antigenic diversity of Plasmodium falciparum and antibody-mediated parasite neutralization," Scandinavian Journal of Immunology, vol. 52, no. 3, pp. 233-239, 2000.

[8] D. Eisen, H. Billman-Jacobe, V. F. Marshall, F. Dave, and F. Coppel, "Temporal variation of the merozoite surface protein 2 gene of Plasmodium falciparum," American Society for Microbiology, vol. 66, pp. 239-246, 2003.

[9] S. Viriyakosol, N. Siripoon, C. Petcharapirat et al., "Genotyping of Plasmodium falciparum isolates by the polymerase chain reaction and potential uses in epidemiological studies," Bulletin of the World Health Organization, vol. 73, no. 1, pp. 85-95, 1995.

[10] I. Felger, L. Tavul, S. Kabintik et al., "Plasmodium falciparum: extensive polymorphism in merozoite surface antigen 2 alleles in an area with endemic malaria in Papua New Guinea," Experimental Parasitology, vol. 79, no. 2, pp. 106-116, 1994.

[11] Z. Premji, P. Ndayanga, C. Shiff, J. Minjas, P. Lubega, and J. MacLeod, "Community based studies on childhood mortality in a malaria holoendemic area on the Tanzanian coast," Acta Tropica, vol. 63, no. 2-3, pp. 101-109, 1997.

[12] C. V. Plowe, A. Djimde, M. Bouare, O. Doumbo, and T. E. Wellems, "Pyrimethamine and proguanil resistance-conferring mutations in Plasmodium falciparum dihydrofolate reductase: polymerase chain reaction methods for surveillance in Africa," The American Journal of Tropical Medicine and Hygiene, vol. 52, no. 6, pp. 565-568, 1995.

[13] I. Felger, T. Smith, D. Edoh et al., "6. multiple Plasmodium falciparum infections in Tanzanian infants," Transactions of the Royal Society of Tropical Medicine and Hygiene, vol. 93, supplement 1, pp. 29-34, 1999.

[14] F. Mwingira, G. Nkwengulila, S. Schoepflin et al., "Plasmodium falciparum msp1, msp2 and glurp allele frequency and diversity in sub-Saharan Africa," Malaria Journal, vol. 10, article 79, 2011.

[15] R. Ibara-Okabande, F. Koukouikila-Koussounda, M. Ndounga et al., "Reduction of multiplicity of infections but no change in msp2 genetic diversity in Plasmodium falciparum isolates from Congolese children after introduction of artemisinincombination therapy," Malaria Journal, vol. 11, article 410, 2012.

[16] M. Ho, H. K. Webster, S. Looareesuwan et al., "Antigen-specific immunosuppression in human malaria due to Plasmodium falciparum," Journal of Infectious Diseases, vol. 153, no. 4, pp. 763-771, 1986.

[17] T. Scorza, S. Magez, L. Brys, and P. De Baetselier, "Hemozoin is a key factor in the induction of malaria-associated immunosuppression," Parasite Immunology, vol. 21, no. 11, pp. 545-554, 1999.

[18] I. Soulama, I. Nébié, A. Ouédraogo et al., "Plasmodium falciparum genotypes diversity in symptomatic malaria of children living in an urban and a rural setting in Burkina Faso," Malaria Journal, vol. 8, article 135, 2009.

[19] H.-P. Beck, I. Felger, W. Huber et al., "Analysis of multiple Plasmodium falciparum infections in Tanzanian children during the phase III trial of the malaria vaccine SPf66," Journal of Infectious Diseases, vol. 175, no. 4, pp. 921-926, 1997.

[20] C. N. M. Mbogo, R. W. Snow, C. P. M. Khamala et al., "Relationships between Plasmodium falciparum transmission by vector populations and the incidence of severe disease at nine sites on the Kenyan coast," The American Journal of Tropical Medicine and Hygiene, vol. 52, no. 3, pp. 201-206, 1995.

[21] S. Kyes, R. Harding, G. Black et al., "Limited spatial clustering of individual Plasmodium falciparum alleles in field isolates from coastal Kenya," American Journal of Tropical Medicine and Hygiene, vol. 57, no. 2, pp. 205-215, 1997.

[22] H. Contamin, T. Fandeur, S. Bonnefoy, F. Skouri, F. Ntoumi, and O. Mercereau-Puijalon, "PCR typing of field isolates of Plasmodium falciparum," Journal of Clinical Microbiology, vol. 33, no. 4, pp. 944-951, 1995.

[23] F. Robert, F. Ntoumi, G. Angel et al., "Extensive genetic diversity of Plasmodium falciparum isolates collected from patients with severe malaria in Dakar, Senegal," Transactions of the Royal Society of Tropical Medicine and Hygiene, vol. 90, no. 6, pp. 704711, 1996.

[24] J. Rono, F. H. A. Osier, D. Olsson et al., "Breadth of antimerozoite antibody responses is associated with the genetic diversity of asymptomatic Plasmodium falciparum infections and protection against clinical malaria," Clinical Infectious Diseases, vol. 57, no. 10, pp. 1409-1416, 2013. 

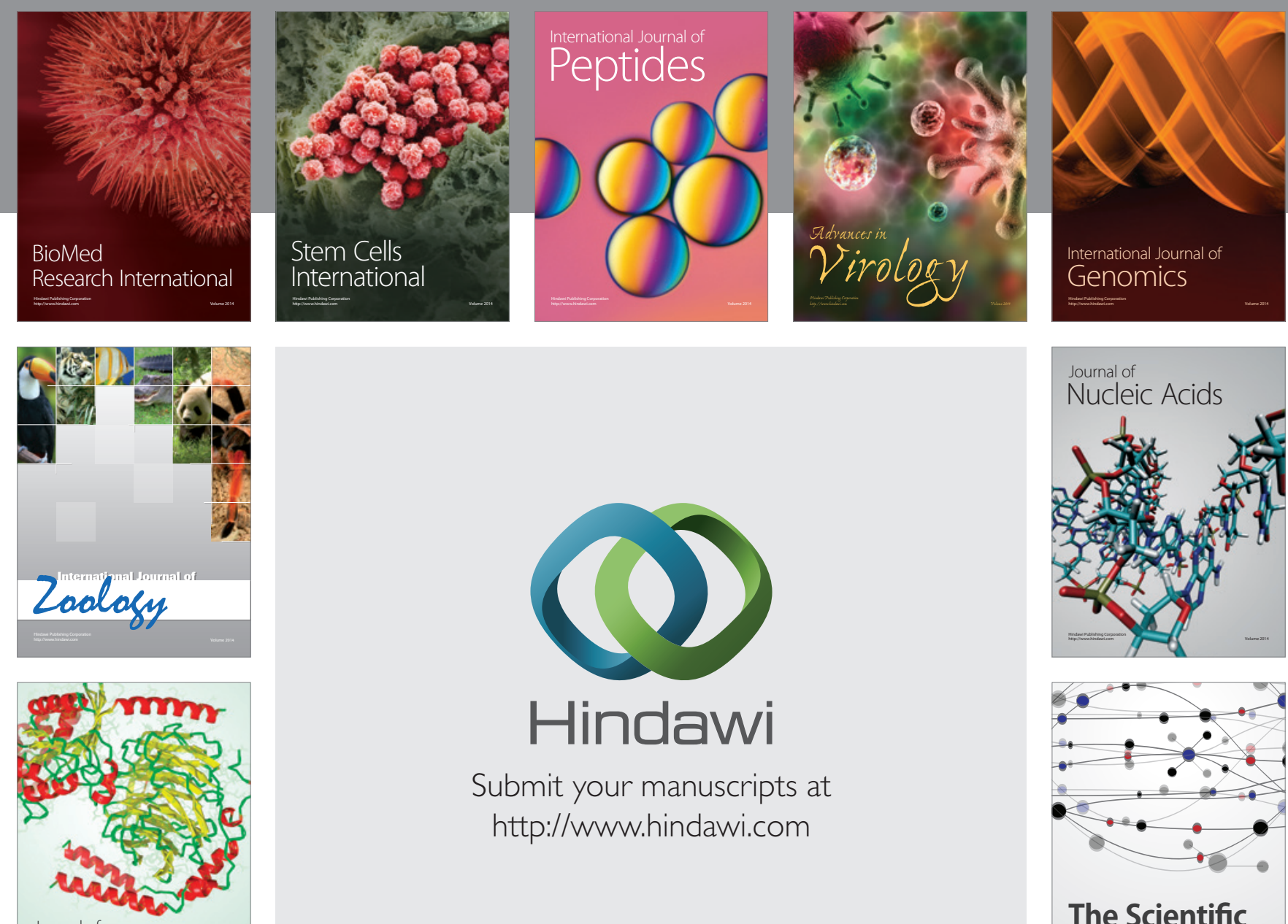

Submit your manuscripts at

http://www.hindawi.com

Journal of
Signal Transduction
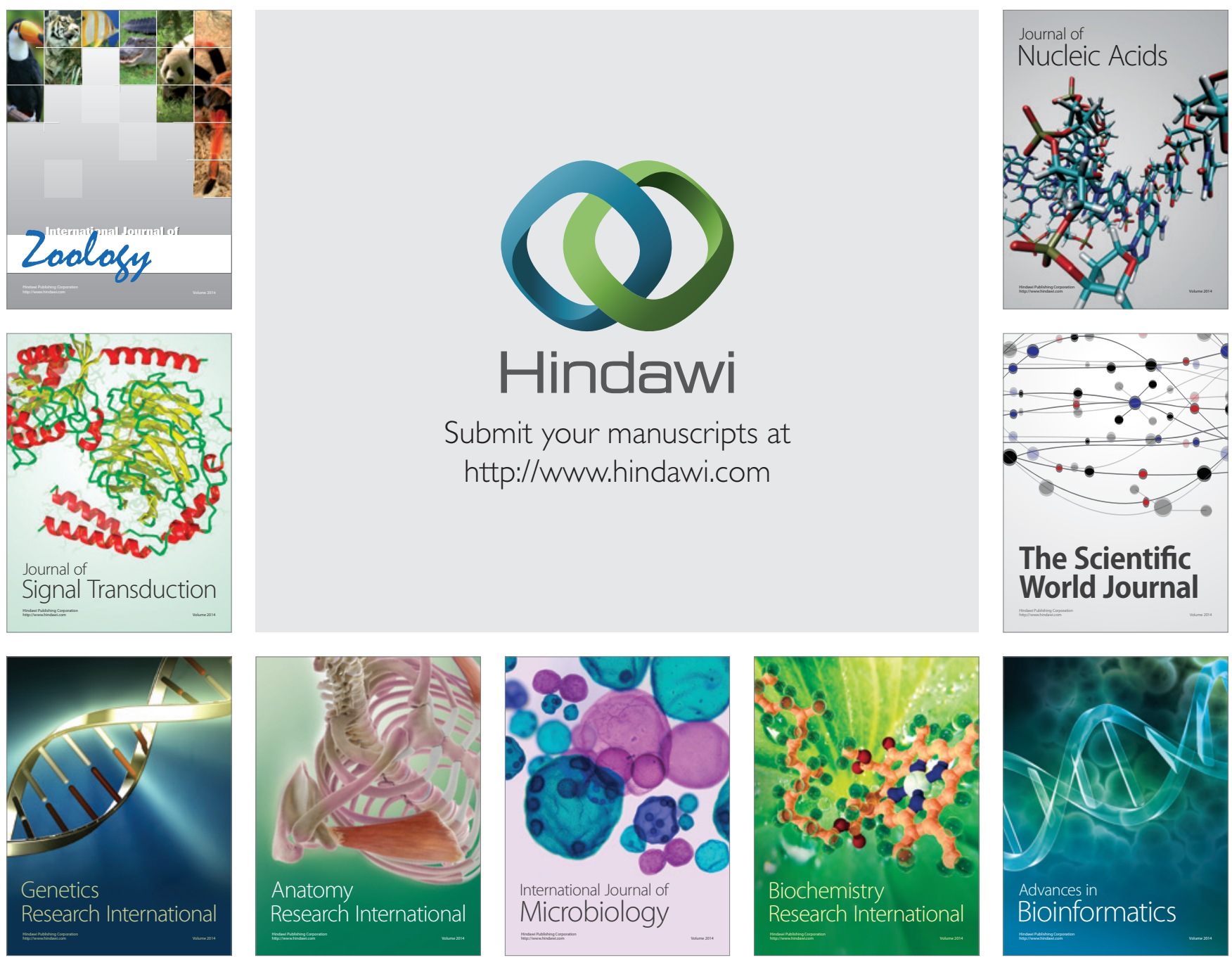

The Scientific World Journal
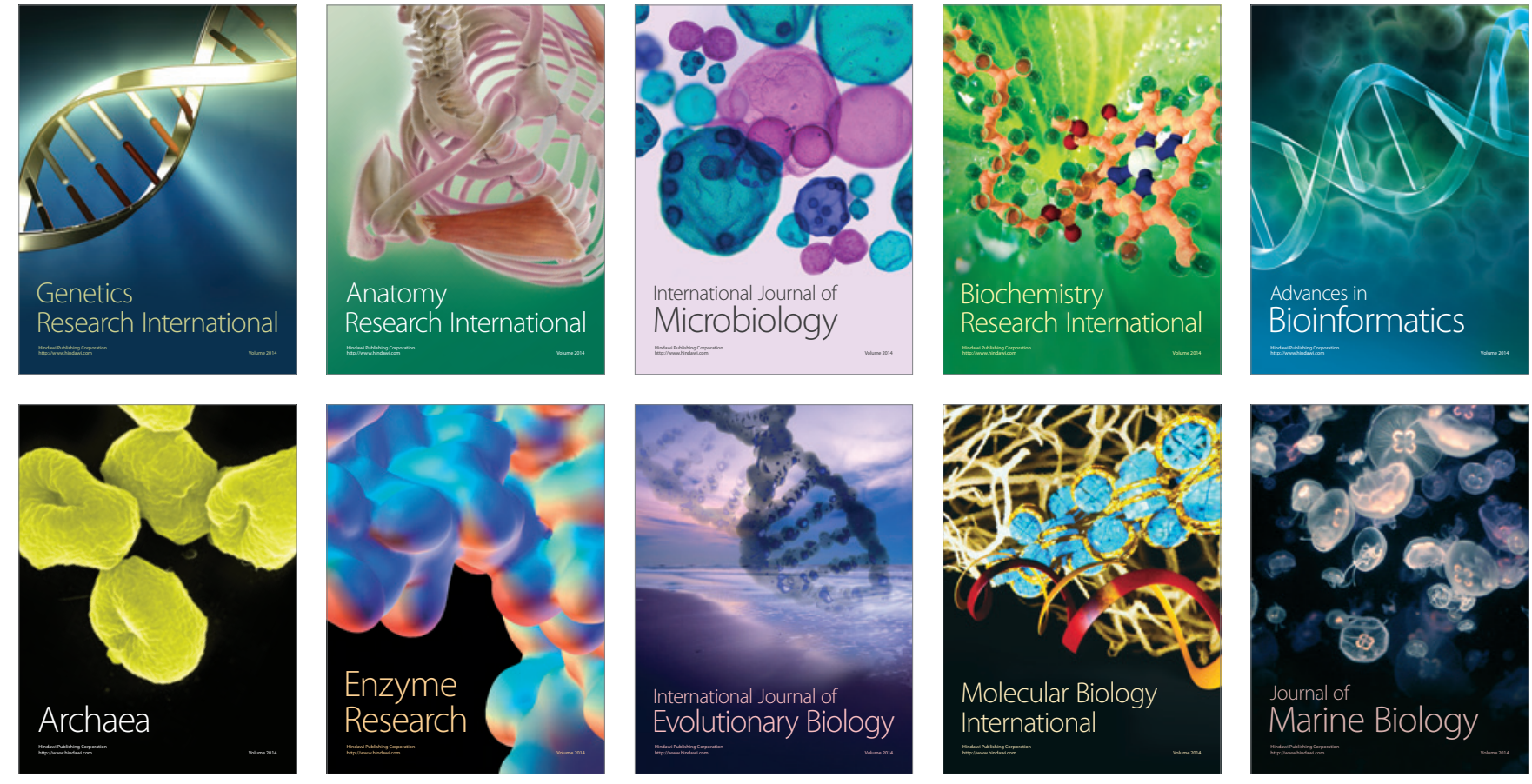